\title{
William Kinderman, Joseph E. Jones (dir.), Genetic Criticism and the Creative Process: Essays from Music, Literature and Theater, 2009
}

Patrizia Metzler

\section{(2) OpenEdition \\ Journals}

Édition électronique

URL : http://journals.openedition.org/genesis/423

DOI : $10.4000 /$ genesis. 423

ISSN : 2268-1590

Éditeur :

Presses universitaires de Paris Sorbonne (PUPS), Société internationale de génétique artistique littéraire et scientifique (SIGALES)

\section{Édition imprimée}

Date de publication : 10 octobre 2010

Pagination : 171-172

ISBN : 978-2-84050-711-6

ISSN : 1167-5101

\section{Référence électronique}

Patrizia Metzler, « William Kinderman, Joseph E. Jones (dir.), Genetic Criticism and the Creative Process: Essays from Music, Literature and Theater, 2009 », Genesis [En ligne], 31 | 2010, mis en ligne le 18 juin 2013, consulté le 22 septembre 2020. URL : http://journals.openedition.org/genesis/423 ; DOI : https://doi.org/10.4000/genesis.423 
À son tour, Rudolf Mahrer met en cause l'opposition du texte au brouillon, en révisant les critères classiques de la textualité. Le critère de cohérence peut s'appliquer au brouillon dès lors que son unité énonciative est reconnue par une " appréhension interprétative ». Celui de sa fonction communicationnelle est relativisé par « la nature intrinsèquement dialogale de l'énonciation » dont témoigne le caractère autodialogique (Bakhtine) de l'écriture. De même, les substitutions (Lebrave) s'apparentent à des reformulations énonciatives. De là, pour Mahrer, la pertinence d'une construction théorique qui permet d'analyser les faits de production (ou d'énonciation) indépendamment des sémiotiques qui les supportent. Cette thèse est illustrée par des exemples empruntés aux manuscrits de Ramuz et qui sont, en effet, démonstratifs, même s'ils prêtent parfois à surinterprétation : la rature d'une seule lettre suffit-elle pour établir que la " difficulté à nommer est ainsi portée sur la scène énonciative $[\ldots]$ », ou bien, des reformulations (textuelles) comme « je la fis s'asseoir » en «Ou plutôt elle s'assit d'elle-même » montrent-elles vraiment « un monde qui résiste au mot» (p. 61-62)?

En fait, ces détails n'ont d'intérêt que pour témoigner, peut-être, d'une différence de sensibilité. Pour le généticien, la complexité du manuscrit, sabré parfois de centaines de ratures, éclaté dans le temps et l'espace, pris dans le réseau enchevêtré d'un dossier, déborde les modèles d'un énoncé textuel (peut-être est-ce un sentiment de « littéraire »?). Mais inversement, Mahrer a certainement raison d'inviter à une réflexion sur la notion de trace et, de façon plus générale, sur l'intérêt, pour la génétique, à réfléchir « sur l'horizon d'interprétabilité de [son] objet - ce qui constituerait un complément épistémologiquement bienvenu à une définition trop "choisiste" de la discipline ». Entre textualité et genèse, le départ est en tout cas donné à un débat neuf.

Les trois contributions suivantes offrent un panorama des nouveaux outils de la recherche génétique. Valentina Chepiga conjugue trois méthodes pour trai- ter, en bonne épistémologie, d'un problème dont la solution est connue d'avance : celle de l'identité des auteurs Romain Gary et Émile Ajar. Entre une confrontation statistique des paramètres syntaxiques qui caractérisent les deux corpus et une étude des variantes génétiques, dont le logiciel MEDITE permet la comparaison automatisée, elle place une description comparative des éléments matériels des deux dossiers. Une analyse des écritures fournirait sans doute un élément d'identification plus décisif encore que celle des documents. Mais l'association de l'analyse matérielle à l'analyse textuelle constitue en soi une prometteuse première méthodologique.

Avec Claire Doquet-Lacoste et Christophe Leblay, le rideau se lève sur le champ tout neuf d'une linguistique de la genèse numérique. Dans ce royaume enchanté, des enregistrements online nous révèlent tout ce que nous avions toujours voulu savoir sur la temporalité de l'écrit. Il est vrai que ce n'est plus tout à fait le même : l'environnement sémiotique (et sans doute pour une part cognitif) de l'écriture a changé. Doquet-Lacoste se demande en quoi ce changement influence les catégories classiques de la génétique (ajout/suppression, variante immédiate/ variante tardive, etc.). Mais elle s'intéresse surtout à ce que l'information temporelle dit désormais sur le comportement et donc sur la subjectivité du sujet écrivant. Cette étude, qu'elle conduit avec beaucoup de finesse dans le cadre de « l'énonciation au sens restreint $»$ (Cullioli), illustre la contribution originale de la recherche online à l'intelligence des faits d'écriture. L'enjeu affirmé est de « $[\ldots]$ construire, à partir de la mise en évidence des logiques d'écriture singulières, les traits distinctifs de l'énonciation scripturale ».

Christophe Leblay propose également (p. 166) une classification spécifique des opérations enregistrées par l'ordinateur, mais ceci dans une visée pédagogique : en revoyant les enregistrements de leurs propres rédactions, les étudiants peuvent progresser dans la pratique de la langue et de l'écriture. Il faut insister sur l'intérêt de ces nouveaux apports à l'étude de la production écrite - d'autant, peut-être, que nous ignorons si le clavier, à son tour, restera toujours à notre disposition.

Genetic Criticism and the Creative Process: Essays from Music, Literature and Theater, dir. William Kinderman et Joseph E. Jones, Rochester, University of Rochester Press, 2009.

\section{Compte rendu par Patrizia Metzler}

Les communications figurant dans ce volume ont été présentées au symposium international intitulé « La critique génétique dans un contexte interdisciplinaire : littérature, arts visuels, théâtre, musique », organisé avec le concours du CNRS par l'université de l'Illinois à UrbanaChampaign en mars 2007. Cette publication témoigne d'un remarquable effort pour faire travailler ensemble des chercheurs issus de disciplines différentes sur le processus de création. Dans son introduction au volume, William Kinderman développe l'idée fondamentale selon laquelle l'approche génétique d'une œuvre d'art améliore la compréhension que nous en avons et ouvre des perspectives inédites. Outre une présentation commentée des différentes contributions au volume, Kinderman illustre par des exemples comment la méthode génétique révèle les influences croisées qui éclairent de multiples aspects du processus de composition. Il présente en particulier plusieurs exemples attestant que dans leur pratique de compositeurs, Wagner aussi bien que Beethoven étaient traversés d'influences extérieures au monde de la musique, et il appelle de ses vœux une intégration de toutes les sources et méthodologies existantes afin de faire progresser les recherches sur le processus de création.

La première partie, intitulée « Textes, variantes et variations : contextes évolutifs en littérature et au théâtre », regroupe cinq contributions témoignant de l'apport de la critique génétique dans cinq domaines de recherche. 
Geert Lernout affirme que l'étude de la genèse d'une œuvre s'inscrit dans le prolongement des recherches classiques menées dans les domaines traditionnels de la bibliographie matérielle, des études textuelles et de la théorie de l'édition. Pour souligner cette continuité, il propose de remplacer le terme de "critique génétique » par celui de «philologie radicale », tout en reconnaissant le caractère réellement novateur de la critique génétique. Il s'attache tout particulièrement à souligner les mérites des études textuelles des textes religieux et à dénoncer les effets négatifs de celles-ci lorsqu'elles sont détournées à des fins idéologiques.

L'article de Daniel Ferrer propose une analogie entre la notion de variante et celle de variation. Alors que le terme de variante renvoie à un «choix entre des éléments considérés comme équivalents », les musiciens parlent de variations pour identifier " des éléments similaires mais différents, juxtaposés dans l'espace ou dans le temps ». Traiter la variante comme une variation implicite permet de « revivifier et de rajeunir » la notion et contribue à une meilleure définition de l'avant-texte.

L'avant-texte du Plaisir du texte illustre la méthode de composition par soustraction de Roland Barthes. L'analyse d'Armine Kotin Mortimer s'attaque au souhait manifesté par Barthes de détruire le texte supprimé. Elle affirme que si nous n'explorons pas toutes les idées, les mots, les phrases rejetés par Barthes, « il n’y aura pas de vérité finale à laquelle nous puissions prétendre ».

La contribution de Jean-Louis Lebrave s'attaque aux problèmes soulevés par les interactions entre la performance et les études de genèse. Posant la question de la possibilité d'études génétiques dans le contexte des arts de la performance, il explore trois mises en scène de l'Électre de Sophocle par Antoine Vitez. La mise en scène est ce qui relie directement le texte à la performance, ainsi qu' aux performances ultérieures pour lesquelles Vitez a réécrit le texte de la pièce. Sachant que le produit final - la performance elle-même - est et doit rester éphémère, Lebrave se demande si les instructions de mise en scène peuvent être traitées comme un avant-texte.

Robert B. Graves discute de l'influence des débuts du cinéma sur les premières œuvres dramatiques de Beckett, et il montre comment les interactions du « pseudocouple " Laurel et Hardy ont eu une influence directe sur l'écriture de Beckett, et ce malgré les dénégations de Beckett lui-même. Graves fait apparaître cette influence en mettant en parallèle des citations extraites des films de Laurel et Hardy et des citations d'En attendant Godot.

Dans la seconde moitié du volume, intitulée « Processus génétiques en musique : de Beethoven à Leroux ", les auteurs abordent les problèmes des pratiques de composition musicale. Les trois premières contributions sont consacrées à Ludwig van Beethoven. Alan Gosman explore les esquisses de Beethoven pour la sonate Waldstein, et il montre comment le compositeur est passé d'esquisses de motifs à l'élaboration des thèmes principaux de cette œuvre. Lewis Lockwood discute deux modèles d'interprétation des esquisses de Beethoven pour le premier mouvement de la Symphonie hérö̈que, l'interprétation «évolutionnaire » et le modèle de «l'image conceptuelle». Pour lui, l'image initiale devient un « invariant » dont l'identité reste stable au cours du processus de composition. Et l'on peut trouver des traces de l'idée initiale dans la version finale. Lockwood met son modèle en rapport avec les notions d'identité et de changement développées par Arnheim, qui considère que la perception d'un objet par un sujet humain est toujours associée dans l'esprit de celui-ci à une perspective particulière. Lockwood rattache ceci au concept de « constances » développé par Arnheim, qui pose qu'une idée musicale va subir des changements tout en conservant des traits qui remontent à sa forme initiale. Peter McCallum analyse le processus de composition dans les derniers quatuors à cordes de Beethoven et met l'accent sur des passages précédant les finals dans lesquels il apparait que le compositeur « compose à haute voix », utilisant des matériaux qui simulent une improvisation afin de préparer l'auditeur à ce qui viendra dans le final. Particulièrement intéressante est l'« exploration de l'autocitation et de l'autoréférence » utilisées par Beethoven pour développer les matériaux destinés à ces passages.

James L. Zychowicz souligne l'importance du travail de chef d'orchestre de Mahler et l'influence marquante de cette activité dans sa pratique de compositeur. Comme Mahler composait le plus souvent pendant l'été, il était nécessaire qu'il conçoive une méthode systématique lui permettant de revenir à son travail de composition en utilisant ses esquisses comme un outil de mémoire.

Joseph Jones s'attaque aux critiques traditionnelles du Chevalier à la rose de Strauss en affirmant qu'une étude critique des esquisses de cette œuvre remet en question les analyses de Kerman et Adorno. L'étude des esquisses lui permet de réfuter l'idée d'une « régression stylistique » attachée à la scène de la présentation de la rose dans l'œuvre de Strauss.

Enfin, Nicolas Donin étudie certains aspects de la composition de Voi(rex) par Philippe Leroux. Grâce à l'interaction avec le compositeur, Donin a pu utiliser une «méthodologie hybride » combinant l'approche génétique et l'anthropologie cognitive afin d'analyser le processus de composition sous tous les angles possibles. Il présente également une application logicielle permettant un recueil exhaustif des données à archiver et à étudier, ainsi que des modalités originales d'exploration de ces données.

Cet ouvrage montre la fécondité d'une approche visant à créer un espace de discussion pour des chercheurs issus de plusieurs disciplines partageant le même désir d'élargir les perspectives critiques dans l'analyse des œuvres relevant de leur domaine. La critique génétique apporte un modèle pour une telle étude élargie, et l'on attend beaucoup de la poursuite des efforts en vue d'instaurer un dialogue entre les chercheurs qui travaillent sur des chefsd'œuvre encore inexplorés. 